\title{
Secure Sharing Between Untrusted Users in a Transparent Source/Binary Deployment Model
}

\author{
Eelco Dolstra \\ Utrecht University, P.O. Box 80089 \\ 3508 TB Utrecht, The Netherlands \\ eelco@cs.uu.nl
}

\begin{abstract}
The Nix software deployment system is based on the paradigm of transparent source/binary deployment: distributors deploy descriptors that build components from source, while client machines can transparently optimise such source builds by downloading pre-built binaries from remote repositories. This model combines the simplicity and flexibility of source deployment with the efficiency of binary deployment. A desirable property is sharing of components: if multiple users install from the same source descriptors, ideally only one remotely built binary should be installed. The problem is that users must trust that remotely downloaded binaries were built from the sources they are claimed to have been built from, while users in general do not have a trust relation with each other or with the same remote repositories.

This paper presents three models that enable sharing: the extensional model that requires that all users on a system have the same remote trust relations, the intensional model that does not have this requirement but may be suboptimal in terms of space use, and the mixed model that merges the best properties of both. The latter two models are achieved through a novel technique of hash rewriting in content-addressable component stores, and were implemented in the context of the Nix system.
\end{abstract}

\section{Categories and Subject Descriptors}

D.2.7 [Software Engineering]: Distribution, Maintenance, and Enhancement

\section{General Terms}

Algorithms, Experimentation, Languages, Management

\section{Keywords}

Software deployment, security, configuration management, source deployment, secure sharing, hash rewriting

\section{INTRODUCTION}

Secure deployment of software is a difficult problem due to trust issues. For instance, how can we trust that binaries that we have

Permission to make digital or hard copies of all or part of this work for personal or classroom use is granted without fee provided that copies are not made or distributed for profit or commercial advantage and that copies bear this notice and the full citation on the first page. To copy otherwise, to republish, to post on servers or to redistribute to lists, requires prior specific permission and/or a fee.

ASE'05, November 7-11, 2005, Long Beach, California, USA.

Copyright 2005 ACM 1-58113-993-4/05/0011 ...\$5.00. downloaded and installed from some remote server are not malicious? Such issues are exacerbated in multi-user environments. If we allow users to install software components on their own, under what circumstances is it safe for other users to use those very same components?

Consider a typical Unix system where components are installed in (say) the directory / usr on Unix or C: \Program Files on Windows. Only the site administrator has the appropriate permissions to do so. This is a highly monolithic security model: the administrator globally makes component selections for all users, along with a determination of whether these components can be trusted. In many environments, this is too inflexible. Consider for instance remote shell servers, shared machines at a web hosting provider, and machines in computational grids. Here there may be many users who need to be able to install software.

Alternatively, individual users can install components in some location where they do have write permission, e.g., /home/alice. But in this case there is no sharing: if another user installs the same software (say, to /home/bob), duplication will occur. This is bad because it increases resource consumption in terms of disk space, disk cache, and network bandwidth.

Also, user-installed software is typically outside of the control of the deployment system (e.g., RPM in the Linux world [3]). This is an important restriction because deployment tools are supposed to track dependencies between installed components. For instance, if Alice installs a component $X$ in her local account that depends on some globally installed component $Y$, then $Y$ should not be removed while $X$ is still present.

A better and more flexible model is one where both administrators and users can just install software from some trusted source such as an operating system distributor, a site-local "channel", or some third-party software vendor — and all such components will end up in a shared component pool, such that components will be shared between users if and only if they are "equal", under some notion of component equality.

That is, users should be logically distinct with regard to the deployment system, i.e., they have the ability to install software themselves; but if a user installs a component previously installed by another user, there will be automatic sharing.

In this paper we explore such a deployment model in the context of the Nix deployment system $[2,1]$. We have previously argued that Nix has many advantages for software deployment:

- Reliable dependencies: we store components in isolation from each other to enable reliable detection of the dependency graph between components, which allows us to prevent missing dependencies.

- Side-by-side deployment of versions and variants: if two components differ in any way (in terms of their build inputs), 
they do not overwrite each other, and therefore the installation of a component can never interfere with the operation of previously installed components.

- Transparent source/binary deployment. Nix is at its heart a source deployment system, like the FreeBSD Ports Collection [4] and Gentoo Linux [5]. Components are deployed through Nix expressions that describe how to automatically build components from source, which is a convenient and flexible deployment model. Source builds are slow, however, but Nix can regain the efficiency of binary deployment in a transparent way by downloading pre-built binaries from remote repositories automatically.

- Safe and automatic garbage collection of unused components.

- Separation of installation and activation: users can have different views on the set of installed components.

The latter point appears to make Nix ideal for multi-user environments, except that until now it lacked a security model. In particular, transparent source/binary deployment requires that when we download a binary from a remote server instead of building from source, we must trust that that binary has actually been built from the same source and has not been tampered with. However, the Nix model assumes that all binaries resulting from a source build action are interchangeable, and allows only one such binary to be present in the system at the same time. Thus, the trust relation with remote servers must be the same for all users, which is not the case in general. (Indeed, some local users themselves might not be trustable.) That is, all users with installation rights must trust each other not to install Trojan horses (malicious components masquerading as legitimate software) or other "malware".

In this paper we improve the Nix system by developing a security model that allows users to safely share installed components. The contributions of this model are the following:

- A transparent source/binary model that supports secure and automatic sharing between users if they are installing the same binaries, or if they have a mutual trust relation. Sharing is enabled through the use of a content-addressable component store, which stores each component under a file name that is a cryptographic hash of the contents of the component.

- We show how content-addressable stores can deal with selfreferential components (components that contain their own file name) through the technique of hash rewriting.

- The resulting model liberates us from the monolithic security assumptions implicit in most deployment systems (e.g., RPM). At the same time, it enables sharing which is absent from non-monolithic models (e.g., Mac OS X application bundles).

- We show how we can have unconditional sharing in a purely source-based deployment model.

This paper is organised as follows. In Section 2 we give an overview of the previous Nix model, and give its semantics. We show in Section 3 that in that model we can at least obtain secure sharing of locally built components. The main contribution is in Section 4, where we describe the new content-addressable model that allows sharing between untrusted users. We extend it in Section 5 by improving sharing between mutually trusted users. We discuss further advantages of the new model in Section 6, and related work in Section 7.

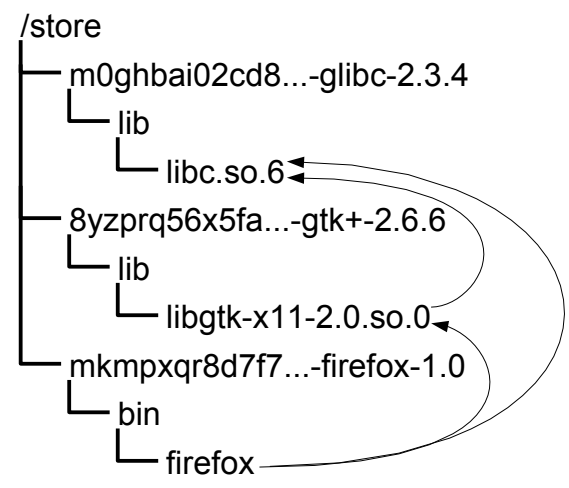

Figure 1: Nix store

\section{THE EXTENSIONAL MODEL}

\subsection{Overview}

In this section we describe a simplified and more powerful version of the model described in $[2]^{1}$, which we here refer to as the extensional model for reasons explained below.

Nix obtains its main advantages - reliable dependencies, sideby-side deployment of versions and variants, and separated component installation and activation - by storing components in isolation from each other in a Nix store, which is simply a directory in the file system that contains components. Figure 1 shows an instance of the Mozilla Firefox component (a web browser) with some of its runtime dependencies (the GUI library GTK, and the $\mathrm{C}$ library Glibc). Each component, which can be a single file or a whole directory tree, has a name - its store path - that uniquely identifies the component, e.g., mkmpxqr8d7f7...-firefox1.0. The prefix is a base-32 representation of a 160-bit cryptographic hash [10] of all inputs involved in building the component, such as sources and dependencies. Thus, any change to the inputs yields a new component with a new store path.

The use of hashes in paths enables reliable identification of dependencies in two ways. First, it prevents undeclared build-time dependencies, for if a store path is not explicitly declared as an input to the build process, tools such as compilers and linkers will not find it (in contrast to "global" directories such as /usr/lib).

Second, it allows us to discover runtime dependencies by scanning for the hash parts of store paths inside components [2]. For instance, Unix executables contain a dynamic library search path (the RPATH [13]) by which the dynamic linker can find libraries. E.g., the Firefox executable in /store/mkmpxqr8d7f7...-firefox$1.0 / \mathrm{bin} /$ firefox contains in its RPATH the path to its GTK runtime dependency, /store/8yzprq56x5fa...-gtk+-2.6.6/lib. By scanning for the hash part 8yzprq56x5fa... inside files of the Firefox component, we discover that it has a dependency on a specific instance of GTK 2.6.6. This technique is generic; Nix knows nothing about the format of Unix executables specifically.

Full information about the runtime dependency graph allows safe deployment by always deploying closures of paths under the dependency relation discovered by scanning. For instance, if we deploy our Firefox instance, we must also deploy the instances of GTK and Glibc (the C library) thus found.

Users can have different views on the set of installed applications

\footnotetext{
${ }^{1}$ The main difference is the removal of explicit closure representations from the Nix store; the references graph is now maintained per path in a database.
} 


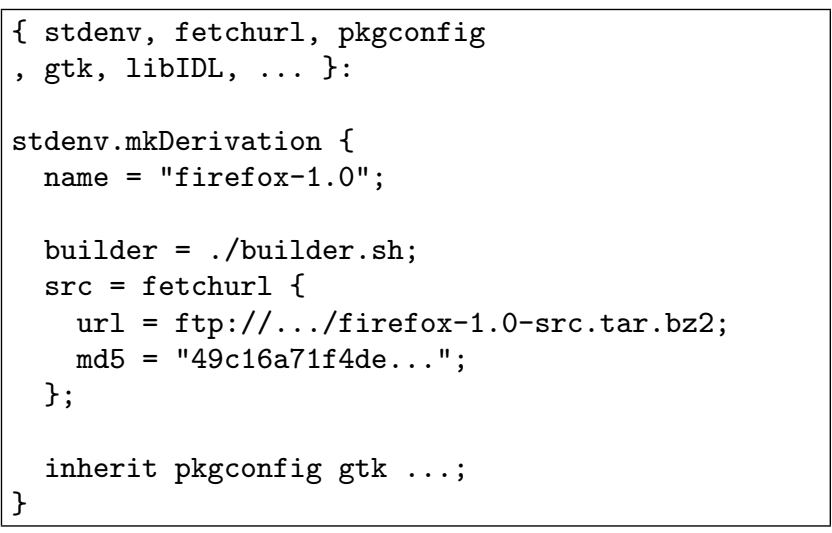

Figure 2: firefox.nix: Nix expression for Firefox

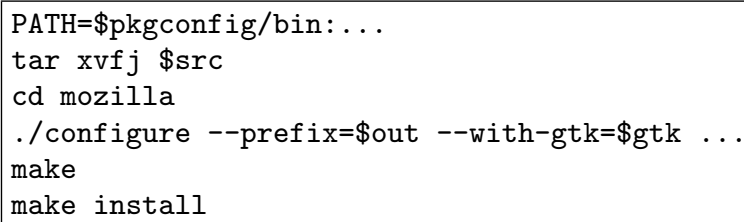

Figure 3: builder.sh: Builder for Firefox

through user environments, which are simply sets of symbolic links [11] to the programs of the components that each user has selected.

\subsection{Nix expressions}

Nix is at heart a source deployment system, meaning that component deployers distribute to client machines Nix expressions that specify how to automatically build components and their dependencies from source. The Nix expression language is a simple functional language that is used to define how to build components and how to compose them. The basic values of the language are strings, paths such as ./builder.sh, and attribute sets of the form $\left\{x_{1}=e_{1} ; \ldots x_{n}=e_{n} ;\right\}$, binding the value of expressions $e_{i}$ to fields $x_{i}$. The form $\left\{x_{1}, \ldots, x_{n}\right\}: e$ defines a function with body $e$ that takes as argument an attribute set with fields named $x_{1}, \ldots, x_{n}$. A function call $e_{1} e_{2}$ calls the function $e_{1}$ with arguments specified in the attribute set $e_{2}$.

An example of a Nix expression to build Firefox is shown in Figure 2, which is a function that specifies how to build Firefox if certain arguments are supplied, namely, Firefox's dependencies. The function arguments are specified at the top, i.e., stdenv, gtk, and so on; these all represent dependencies. (The dependency stdenv provides a standard Unix build environment, i.e., a C compiler, common Unix tools, and so on.) When the function is called with concrete arguments for these dependencies, a derivation is returned, which represents a component build action. The derivation is produced by the call stdenv.mkDerivation. The arguments of this call are the inputs to the build process. The construct inherit causes an argument to be inherited from the surrounding lexical scope, so, e.g., the gtk function argument is passed verbatim as a derivation input. The function fetchurl downloads the source of Firefox from the Internet and checks that the downloaded content matches the specified cryptographic hash.

The special argument builder identifies a script that performs the actual build. Figure 3 shows the builder for Firefox, which is a fairly standard command sequence to build a Unix component. All derivation arguments specified in the call to stdenv.mkDerivation

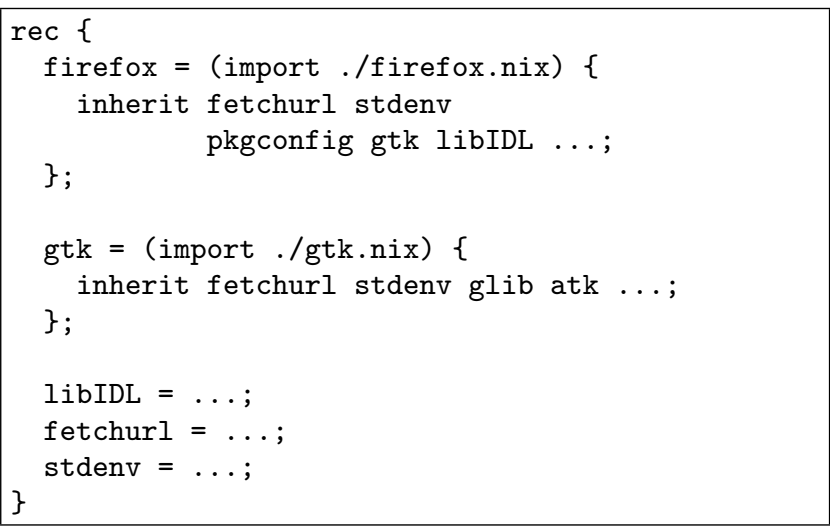

Figure 4: composition.nix: Nix expression composing Firefox, GTK, etc.

are passed as environment variables. In the case of arguments that denote dependencies, such as gtk, these environment variables hold the paths of those dependencies in the Nix store. For instance, the environment variable gtk will hold the path of the GTK instance (e.g. /store/8yzprq56x5fa...-gtk+-2.6.6). Also, the special variable out contains the store path where the builder is to install its output (e.g., /store/mkmpxqr8d7f7...-firefox-1.0). How these paths are computed is described below.

Since the Firefox expression in Figure 2 is a function, to build a concrete Firefox instance we have to call the function, passing in values for the expected arguments. An example of such a call is shown in Figure 4. The Firefox expression in Figure 2 is imported from firefox.nix and called with arguments that are inherited from the lexical scope. Like Firefox, these values (e.g., gtk) are computed by importing Nix functions and calling them with the appropriate arguments. So a value like gtk also evaluates to a call to stdenv.mkDerivation that builds the component using its own particular builder, sources, dependencies, etc. The rec construct causes attributes to be mutually recursive, e.g., firefox can refer to gtk.

To automatically install a component from source, users obtain Nix expressions, and run a command such as

$$
\text { \$ nix-env -f composition.nix -i firefox }
$$

The command nix-env evaluates the firefox value in Figure 4, recursively builds all components, and makes sure that the programs of the resulting top-level component (e.g., /store/mkmpxqr8d7f7...firefox-1.0/bin/firefox) are added to the user's PATH environment variable.

\subsection{Store derivations}

Nix expressions are not built directly; rather, they are translated to the more primitive language of store derivations, which encode single component build actions. Store derivations are placed in the Nix store, and as such have a store path too. The advantage of this two-level build process is that the paths of store derivations give us a way to uniquely identify objects of source deployment, just as paths of binary components allow us to uniquely identify objects of binary deployment.

A store derivation specifies the output path that it builds, the paths of its input derivations (build time dependencies), the paths of its immediate sources (which are copied to the Nix store by the translation process), the path of the builder, and the shell environment to be passed to the builder. For example, the store derivation 
resulting from translating the firefox variable in Figure 2 resides in a store path /store/rax19fjg9691-firefox.drv containing:

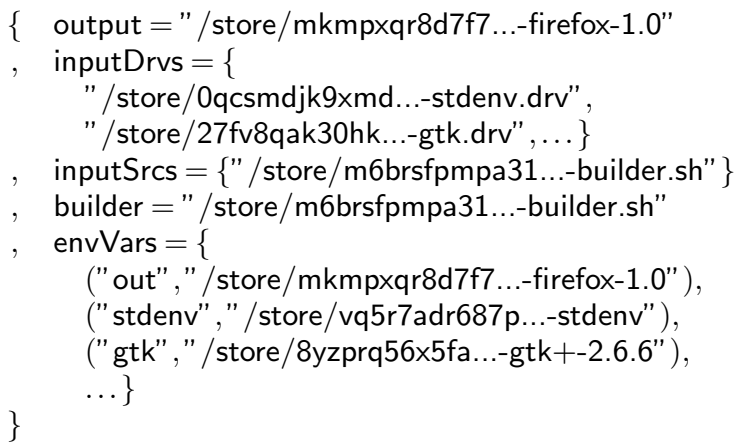

Note that the environment variables refer to paths produced by the input derivations. For instance, the path / store/8yzprq56 55 fa...gtk+-2.6.6 is the output of the derivation /store/27fv8qak30hk...gtk.drv.

How are the hash parts of store paths computed? For sources and store derivations, they are the hash of the content of the file. That is, when copying a source file at path $p$ to the Nix store, the hash is

$$
\text { hash(" src:" + contents }(p) \text { ) }
$$

where the function contents $(p)$ computes a canonical serialisation of the file system contents at path $p$ (i.e., a dump of $p$ ), hash $(s)$ returns a base-32 representation of a 160-bit cryptographic hash of string $s$, and + denotes string concatenation. In effect, those parts of the Nix store representing copied sources can be said to be content-addressable: if we know the content of a file, we also know its path.

On the other hand, for output paths (the results of derivations), we do not know the contents of a component until after it has been built - but we have to assign it a path before it is built! This is because Unix components for instance typically store references to their own installation path into executables, libraries, and other files. Therefore we compute the hash part of the output of a derivation $d$ as a hash of the input derivations:

$$
\text { hash(" out:" +show } \left.\left(d^{\prime}\right)\right)
$$

where $d^{\prime}$ equals $d$ with output set to the empty string, and show $(d)$ yields a string representation of the derivation $d$. The prefixes "src:" and "out:" are used to prevent sources and output paths from accidentally hashing to the same path.

\subsection{Transparent Source/Binary Deployment}

The model as described above is a source deployment model: component distributors deploy to clients Nix expressions that describe how to automatically build the components from source by running build actions. That is, Nix expressions essentially form a high-level Makefile for components. This is the model used by source-based deployment systems such as the FreeBSD Ports Collection [4] and Gentoo Linux [5]. Such a model is convenient and flexible for developers and component distributors, because it obviates the need to explicitly make binary packages, and because Nix expressions can express component variability, allowing customisation of components. On the other hand, source deployment is bad for end-users, because it is slow: installing Firefox from Figure 4 entails building not only Firefox but also all its dependencies, e.g., the $\mathrm{C}$ compiler and library, GUI libraries, and so on.

For this reason Nix has the notion of transparent source/binary deployment through its substitute mechanism. Distributors can prebuild specific instances of Nix expressions and make them centrally available, e.g., on a web server. Clients can then register the fact that such pre-built components are available, i.e., that if we subsequently wish to build a store path $p$, we can do so by downloading a binary from URL $u$ instead of building. Thus, source deployment transparently optimises into binary deployment. Moreover, if the user locally modifies Nix expressions or sources (say, to optimise for a specific environment), this might cause the output paths of the derivations to change, in which case binary deployment automatically falls back to source deployment.

\subsection{Semantics}

Here we formalise some aspects of the semantics of the extensional model, as a basis for the discussion of the intensional model in Section 4.

Nix maintains some meta-information about store paths in a few database tables. The set valid: $\{$ Path $\}$ (where Path in the universe of store paths) lists the paths that have been successfully built, added as a source, or obtained through a substitute. It does not include paths that are currently being built, or that have been left over from failed operations. (We write valid $[p]$ to denote that a path is valid.)

The mapping references : Path $\rightarrow\{$ Path $\}$ maintains the dependency graph, i.e., the set of store paths referenced by the contents of each store path as discovered by scanning for hashes. At any time Nix maintains the closure invariant:

$$
\forall p: \operatorname{valid}[p] \rightarrow \forall p^{\prime} \in \operatorname{references}[p]: \operatorname{valid}\left[p^{\prime}\right]
$$

This means that the set of valid paths is closed under the references relation. The closure of a path $p$ is the set of all paths that might be accessed due to the execution of the component $p$ :

$$
\operatorname{closure}(p)=\{p\} \cup \bigcup_{p^{\prime} \in \text { references }[p]} \operatorname{closure}\left(p^{\prime}\right)
$$

The mapping substitutes : Path $\rightarrow$ (Path, [String]) stores the substitutes that have been registered by users. The right-hand side is the name of a program with its command-line arguments that should be executed to obtain the contents for the path denoted in the left-hand side, e.g., ("download-url.sh", [" http://...", ...]).

The following invariant states that references must be known for valid paths, as well as for paths that are invalid but have substitutes.

$$
\forall p:(\operatorname{valid}[p] \vee \text { substitutes }[p] \neq \varepsilon) \rightarrow \operatorname{references}[p] \neq \varepsilon
$$

(The special value $\varepsilon$ indicates that no entry occurs for a value in the given mapping.) It is necessary to know the references of substitutable paths in order to maintain the closure invariant on deployment. That is, prior to installing a path through a substitute, we must first build or download its references. For instance, from Figure 1 it follows that prior to downloading Firefox we must download Glibc and GTK (in that order).

Figure 5 shows the build algorithm for derivations in pseudocode. The operator $\leftarrow$ denotes assignment, and $x \longleftarrow y$ is shorthand for $x \leftarrow x \cup y$. Locking of output paths to prevent simultaneous builds of a derivation is omitted in this paper (though not in our implementation). Given a store derivation $d$, the algorithm builds the output by running the builder, but only if the output was not already valid and could not be made valid through a substitute. If we do build, we subsequently scan for references to input paths (that is, the union of closures of input sources and of output paths of input derivations), and set the path to valid. Not shown here is that the implementation maintains the invariants at all times by wrapping database operations in transactions as appropriate. 


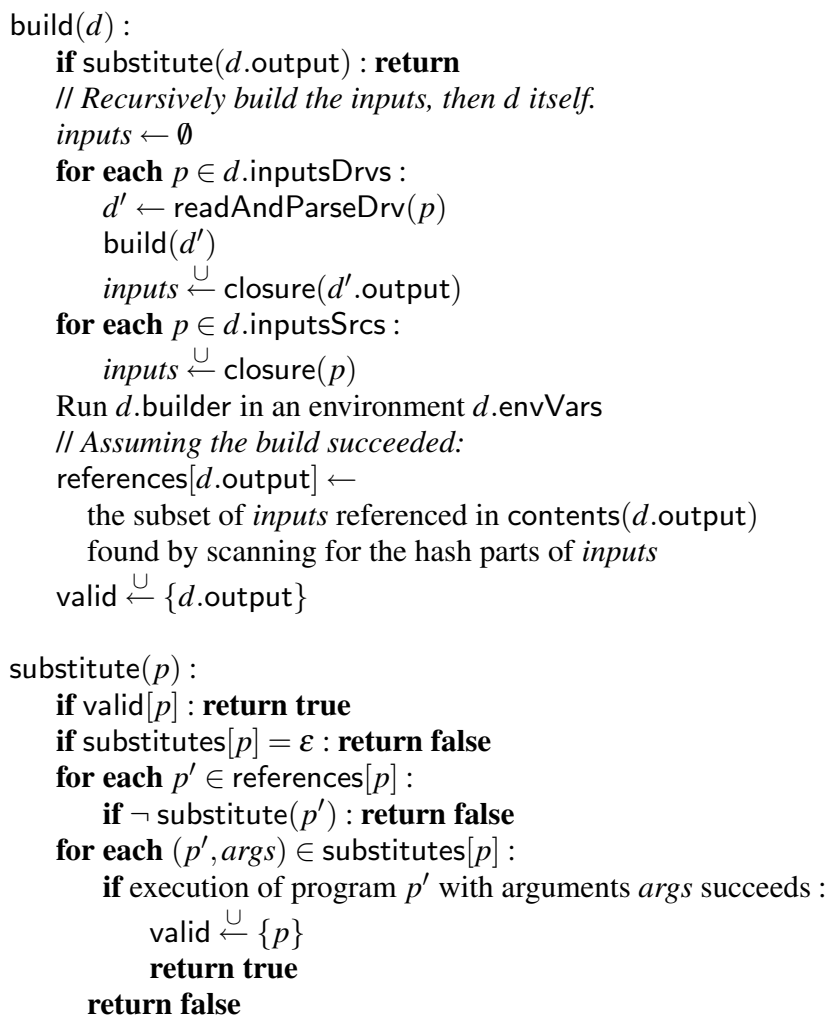

Figure 5: Build algorithm in the extensional model

\subsection{Extensionality}

So why do we call this model extensional? The reason is that we make an assumption of extensional equality. Build operations in general are not pure: the contents that a builder stores in the output path can depend on impure factors such as the system time. For instance, linkers often store a timestamp inside the binary contents of libraries. Thus, each build of a derivation could produce a subtly different output.

However, the model is that such impure influences, if they exist, do not matter in any significant way. For instance, timestamps stored in files generally do not affect their operation. Hence extensional equality: two mathematical objects (such as software components) are considered extensionally equal if they behave in the same way for any operation on them. That is, we do not care about their internal structure.

Thus, while any derivation can have a potentially infinite set of output path contents that can be produced by an execution of its builder, we view the elements of that set as interchangeable.

But this is a model - an assumption about builders. For instance, if a builder yields a completely different component when invoked at a certain time of day, it is beyond the scope of the model. In reality, also, we can always observe inequality by observing the internal encoding of the component, since that is a permissible operation. But the model is that such observations do not take place or do not have an observable effect.

\subsection{Sharing}

Sharing a Nix store in the extensional model is only possible if all users of the Nix store trust each other. For instance, suppose that Alice has obtained a Nix expression $E$ from a trusted source, and pulls substitutes from machine $X$, where $X$ is a malicious ma- chine that provides Trojaned binaries for the output paths of the derivation produced by $E$. This may cause Alice's account to be compromised. If subsequently Bob installs the same expression $E$, but pulls from trusted machine $Y$, he will still obtain the Trojaned binary pulled by Alice. This is because both binaries occupy the same location in the file system, and Nix will not install another substitute if the output path is already valid.

The problem here is that machine $X$ claims that its substitute is an output of some derivation $d$, but it isn't. However, since we have no way to verify such a claim, we cannot discover this fact. We have to trust such a claim, and hence we must have a trust relation with machine $X$.

\section{LOCAL SHARING}

In Section 2.7 we saw that sharing between machines is only possible in the extensional model if all users have the same remote trust relations. For locally-built derivations on the other hand (i.e., when not using substitutes), we can allow mutually untrusted users. The trick is in preventing a user from influencing the build for some derivation $d$ in such a way that the result is no longer a legitimate output of $d$.

For instance, if Alice has direct write access to the Nix store, she can start a build of derivation $d$, then overwrite the output path with a Trojan horse. Similarly, even if builds are done through a server process that executes builds on behalf of users but running under a different user ID (uid), Alice can interfere with the build of $d$ by starting a build of a specially crafted derivation $d^{\prime}$, the builder of which writes a Trojan horse to the output path of $d$.

We can prevent this as follows. First, users no longer have direct write access to the Nix store. All builds are performed by a Nix server process on behalf of users. The server runs builders under uids that are distinct from those of ordinary user or system processes (e.g., nix-build- $\{1,2, \ldots\}$ ). Also, no two concurrent builds can have the same uid. This prevents one builder from interfering with the output of another, as illustrated above. Thus, the server maintains a "pool" of free and in-use uids that can be used for building.

When a build finishes, prior to marking the output path as valid, we do the following:

- Ensure that there are no processes left running under the uid selected for the builder. On modern Unix systems this can be done by performing a kill(-1, SIGKILL) operation while executing under that uid, which has the effect of sending the KILL signal to all processes executing under uid.

- Change ownership of the output to the global Nix user.

- Remove write permission and any set-user-ID and set-groupID bits (which are special permission bits on files that cause them to be executed with the rights of a different user or group - a potential security risk).

Note that the latter two steps have a subtle race condition. For instance, if we change ownership first, we have the risk of inadvertently creating a setuid binary owned by the global Nix user. If however we remove write and setuid permission first, a left-over process spawned by the builder could restore those permissions $b e$ fore the ownership is changed. This is why the first step is important. Also, on Unix, if a left-over process opened a file before the ownership changes, it can still write to it after the change, since permissions are only checked when a file is opened.

In conclusion, we can securely do source deployment in the extensional model with sharing. Of course, that is not enough: we 
also want to have transparent binary deployment through the substitute mechanism.

Note that none of this ensures that binary components can be trusted. What it does is ensure that if any user builds a Nix expression, the result will be a binary built by that Nix expression without outside interference. This implies that if the Nix expression is trusted, any locally built binary produced by it is also trusted.

\section{THE INTENSIONAL MODEL}

As we saw in Section 3, we can have secure sharing of locally built derivation outputs, but not of remotely built outputs obtained through the substitute mechanism. All users have to trust that the contents produced on another machine purportedly from some derivation $d$ is indeed from derivation $d$. As stated above, such a trust relation must be global for a Nix installation. In this section we develop a substantially more powerful model in which this is not the case. We do this by moving to a fully content-addressable Nix store for all store objects, including derivation outputs. As we shall see, this is not trivial due to self-referential components.

In the example in Section 2.7, Alice and Bob had different trust relations mapping different outputs onto the same store paths. This problem does not exist in a content-addressable store, where the hash component of the store path is equal to the hash of the contents of that path. In such a system content equality implies path equality.

If we have this property, then different users can have different trust relations: for each user we can have a different derivation to output path mapping. This is the intensional model - equality is defined by internal contents, not observable behaviour. This model is much stronger than the extensional model, since it doesn't make the simplifying but unenforcible assumption of builder purity. Rather, intensionality is an inherent property of the system.

\subsection{Content-addressability}

The crucial property of the intensional model is that the Nix store is now content-addressable: if we know (the hash of) the contents of a store object, we know its store path. Formally, this means that the following hash invariant holds:

$$
\forall p: \operatorname{valid}[p] \rightarrow \operatorname{hashPart}(p)=\operatorname{hash}(\operatorname{contents}(p))
$$

where hashPart $(p)$ returns the hash component of path $p$, e.g., for /store/mg12dly8...-firefox-1.0 it returns mg12dly8.... This invariant says that for all valid paths, the hash part of the store path equals the hash of the contents of that store path. For instance, the store path /store/mg12dly8...-firefox-1.0 implies that its content has cryptographic hash mg12dly8....

So how does content-addressability help us to achieve secure sharing in multi-user Nix stores? The answer is that users can now independently install software, i.e., build derivations. If the results of those independent builds are the same, we get sharing; if they differ due to impurity, we do not get sharing. This applies not just to local builds but more significantly to substitutes.

In the example of Section 2.7, when Alice installs a derivation for Firefox using a Trojaned substitute from a malicious machine, the result will end up in some path, say /store/x1cpydjlgxbw...firefox-1.0. If Bob installs the same derivation but using a legitimate substitute, the content will differ and so the result will necessarily be in a different store path, e.g., /store/mg12dly8...-firefox1.0. His user environment will include the latter path. Thus, he is insulated from Alice's bad remote trust relation.

\subsection{Hash rewriting}

The property of content-addressability is easily stated but not so easily achieved. This is because we do not know the content hash of a component until after we have built it, but we need to supply an output path to the builder through the out environment variable beforehand, so that it knows where to store the component.

We solve this problem through hash rewriting. The idea is that we perform a build in a store path $p$ with a randomly generated hash part. Afterwards, we compute the content hash, and rename $p$ to

$$
p^{\prime}=\operatorname{subst}(p,\{\operatorname{hashPart}(p) \rightsquigarrow \operatorname{hash}(\text { contents }(p))\})
$$

(where subst $(s, r)$ is a function that applies a set of substitutions $r$ to the string $s$; substitutions are denoted as $x \rightsquigarrow y$ ). That is, the temporary path is changed to one that obeys the hash invariant. Note that the replacement string has exactly the same length in order not to break binary components (see Section 6.1 for a further discussion of the risks of hash rewriting).

There is a snag, however: simply renaming the temporary path doesn't work in the case of self-references, i.e., if the binary image of a file refers to its own store path. This is quite common. For instance, the RPATH of a Unix executable (mentioned in Section 2.1) frequently points to its own directory so that related library components can be found. If we rename the temporary path $p$ to $p^{\prime}$ in such a case, the references to $p$ will become dangling references, and the component probably will not work anymore.

We might be tempted to replace all occurrences of the string hashPart $(p)$ in the content of the component with hashPart $\left(p^{\prime}\right)$. However, since this changes the content of the component, it also invalidates the hash! And with cryptographic hashes it is not feasible to compute a "fixed point", i.e., a string containing the hash to which the string hashes.

We fix this problem by computing hashes modulo self-references. Essentially, this means that we ignore self-references when computing the hash. First, when computing the hash of contents $(p)$, we zero out all occurrences of the string hashPart $(p)$. That is,

$$
\begin{aligned}
& p^{\prime}=\operatorname{subst}(p,\{\text { hashPart }(p) \rightsquigarrow \\
& \quad \text { hashModulo }(\operatorname{contents}(p), \text { hashPart }(p))\})
\end{aligned}
$$

where hashModulo(s, h) is defined as

$$
\operatorname{hash}\left(\sum_{i \in \operatorname{find}(s, h)}(i+": ")+": "+\operatorname{subst}(s,\{h \rightsquigarrow \mathbf{0}\})\right)
$$

The function find $(s, h)$ yields the offsets of the occurrences of the substring $h$ in the string $s$, and $\mathbf{0}$ denotes a string consisting of binary 0 s of the same length as $h$. It is necessary to encode the offsets of the occurrences of $h$ into the hash to prevent hash collisions for strings that are equal except for having either $h$ or 0 -strings at the same location. The colons simply act as disambiguators, separating the offsets and the contents.

Second, we copy $p$ to $p^{\prime}$, while rewriting all occurrences of hashPart $(p)$ in the contents of $p$ with hashPart $\left(p^{\prime}\right)$ :

$$
\begin{array}{r}
\text { contents }\left(p^{\prime}\right)=\operatorname{subst}(\text { contents }(p), \\
\left.\left\{\operatorname{hashPart}(p) \rightsquigarrow \operatorname{hashPart}\left(p^{\prime}\right)\right\}\right)
\end{array}
$$

Note that

$$
\begin{aligned}
& \text { hashModulo }(\text { contents }(p), \operatorname{hashPart}(p)) \\
= & \text { hashModulo }\left(\text { contents }\left(p^{\prime}\right), \operatorname{hashPart}\left(p^{\prime}\right)\right)
\end{aligned}
$$

even though

$$
\operatorname{hash}(\operatorname{contents}(p)) \neq \operatorname{hash}\left(\operatorname{contents}\left(p^{\prime}\right)\right)
$$

in case of self-references. That is, the hash modulo the randomly generated hash part does not change after rewriting. 
We can now formulate the hash invariant as follows:

$$
\begin{aligned}
& \forall p: \operatorname{valid}(p) \rightarrow \text { hashPart }(p)= \\
& \quad \text { hashModulo }(\text { contents }(p), \operatorname{hashPart}(p))
\end{aligned}
$$

\subsection{Semantics}

We can now formalise the intensional model. The main difference with the extensional model is that output paths are no longer known a priori. But because of this, we cannot prevent re-building a derivation by checking (as was done in Figure 5) whether its output path is already valid. The same applies to checking for substitutes, which are also keyed on output paths.

Also, in the intensional model, due to impurity a single derivation can result in several distinct components residing at different store paths, if the derivation is built multiple times (e.g., by different users). That is, a derivation actually defines an equivalence class of store paths within the Nix store, the members of each class all having been produced by the same derivation. Thus, we add to store derivations a field eqClass : EqClass and remove the field output.

So what is an equivalence class (i.e, what is the type EqClass)? In fact, the equivalence class eqClass is exactly the same as the original output field! It is computed in the same way that the output field was computed in the extensional model: by hashing the derivation with its eqClass field set to the empty string. For instance, the Firefox derivation might have d.eqClass =" / store/mkmpxqr8d7f7...-firefox-1.0".

So we have really just renamed the field output to eqClass. However, there is an important difference in the meaning of eqClass. The equivalence class path is "virtual": it is never built. The reason for using store paths for equivalence classes is that it gives us an easy way to refer to the output of a derivation from other derivations. For instance, the envVars field of a derivation that depends on Firefox must in some way refer to the path of the Firefox component, even though this path is not known in advance anymore. When we build a derivation $d$ depending on derivation $d^{\prime}$, we simply rewrite in $d$.envVars all occurrences of hashPart $\left(d^{\prime}\right.$.eqClass) to a trusted member of the equivalence class denoted by $d^{\prime}$.eqClass. Equivalence classes are computed in exactly the same way

Since we must remember for each derivation what output paths were produced by it and who built or substituted them, we define a database mapping eqClassMembers : EqClass $\rightarrow\{$ (Userld, Path $)\}$ meaning that an equivalence class maps to a set of store paths along with the name of the user that built or substituted the store path. A store path can occur multiple times for different users.

The set of trusted paths in the equivalence class of a derivation output is simply the set of valid or substitutable paths for some user:

$$
\begin{aligned}
& \text { trustedPaths }(\text { eqClass, user })= \\
& \quad\{p \mid(\text { user }, p) \in \text { eqClassMembers }[\text { eqClass }]\}
\end{aligned}
$$

\subsubsection{Equivalence classes and closures}

A path can be a member of multiple equivalence classes. This is easy to see: we can conceive of any number of different derivations that produce the output "Hello World" in various ways. So we cannot unambiguously say to what equivalence class such a path belongs. However, as we shall see below, there are times when we need to know this. In particular, when we compute the closure of a path $p$, we would like to know to what equivalence class each reference belongs. Such a query is only meaningful given a certain context, i.e., when we compute the closure of a path $p$ in an equivalence class $e$.

To this end, we maintain a database mapping refClasses: (Path, Path $) \rightarrow\{($ EqClass, EqClass $)\}$. This table allows us to determine equivalence classes when we are following the references graph. It has the following meaning: if $\left(e, e^{\prime}\right) \in \operatorname{refClasses}\left[\left(p, p^{\prime}\right)\right]$, then there is an edge in the references graph from path $p$ in equivalence class $e$ to path $p^{\prime}$ in equivalence class $e^{\prime}$.

The function closure' $(p, e)$ computes the closure of a path $p$ in equivalence class $e$, yielding not just the paths in the closure but also their equivalence classes; thus, it returns a set of pairs $(p, e)$ : (Path, EqClass).

$$
\operatorname{closure}^{\prime}(p, e)=\{(p, e)\} \cup \bigcup_{p^{\prime} \in \operatorname{references}[p]} \text { followRef }\left(p, e, p^{\prime}\right)
$$

The auxiliary function follow $\operatorname{Ref}\left(p, e, p^{\prime}\right)$ yields the closure of $p^{\prime}$ coming from path $p$ in equivalence class $e$ :

$$
\text { followRef }\left(p, e, p^{\prime}\right)= \begin{cases}\operatorname{closure}^{\prime}\left(p^{\prime}, \varepsilon\right) & \text { if } e s^{\prime}=\emptyset \\ \bigcup_{e^{\prime} \in e s^{\prime}} \operatorname{closure}^{\prime}\left(p^{\prime}, e^{\prime}\right) & \text { otherwise }\end{cases}
$$

where

$$
e s^{\prime}=\left\{e^{\prime} \mid\left(e, e^{\prime}\right) \in \operatorname{refClasses}\left[\left(p, p^{\prime}\right)\right]\right\}
$$

The condition $e s^{\prime}=\emptyset$ is to handle paths that are not in any equivalence class. This is quite normal: paths that are not outputs of derivations (such as sources) need not be in equivalence classes. For such paths, the special value $\varepsilon$ is used to denote the absence of an actual equivalence class.

\subsubsection{Equivalence class collisions}

The fact that a derivation can resolve to any number of output paths due to impurity, leads to the problem that we might end up with a closure that contains more than one element from the output equivalence class of a derivation.

Figure 6 shows an example of this problem. Suppose that Alice has locally built gtk and pkgconfig (which both depend on glibc). She has also registered Bob's remotely built libIDL as a substitute (which also depends on glibc). However, though Bob's glibc was built from the same derivation, due to impurities the build result is different. Thus, eqClassMembers $\left[\right.$ glibc $\left._{e q}\right]=\left\{\right.$ ("alice", glibc $\left._{A}\right)$, ("alice", glibc $\left.\left.c_{B}\right)\right\}$. (For brevity, we use variables glibc ${ }_{A}$, glibc $_{e q}$ and so to represent concrete paths and equivalence classes.) This is in itself not a problem. However, suppose that Alice next tries to build firefox, which depends on gtk, pkgconfig, and libIDL. We then end up with a Firefox binary that links against two glibcs. This might work, or it might not - depending on the exact semantics of dynamic linking. In any case, it is an observable effect - it influences whether a build succeeds and whether the build result works properly.

Thus, we need to prevent that any closure ever contains more than one path from an equivalence class. This is the equivalence class uniqueness invariant:

$$
\begin{aligned}
& \forall p \in \text { Path : valid }[p] \rightarrow \forall e \in \text { EqClass : } \\
& \quad \forall\left(p_{1}, e_{1}\right) \in \text { closure }^{\prime}(p, e): \forall\left(p_{2}, e_{2}\right) \in \text { closure }^{\prime}(p, e): \\
& \quad\left(e_{1} \neq \varepsilon \wedge e_{1}=e_{2}\right) \rightarrow p_{1}=p_{2}
\end{aligned}
$$

That is, for any two elements $\left(p_{1}, e_{1}\right)$ and $\left(p_{2}, e_{2}\right)$ in any closure of a valid path $p$, if the equivalence classes are the same $\left(e_{1}=e_{2}\right)$, then the paths must also be the same $\left(p_{1}=p_{2}\right)$. The condition $e_{1}=\varepsilon$ is to handle paths that are not in any equivalence class (such as sources).

So when we build a derivation, we must select from the paths in the union of input closures one from each equivalence class. However, we must still maintain the closure invariant. For instance, in Figure 6, we cannot just select the set $\left\{\right.$ glibc $_{A}$, gtk , pkgconfig $_{A}$, liblDL $L_{B}$, since libIDL $L_{B}$ depends on glibc $_{B}$ which is not in this set. 


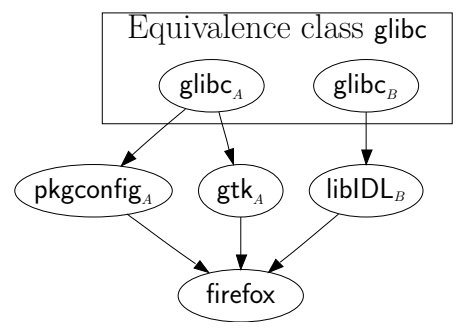

Figure 6: An equivalence class collision

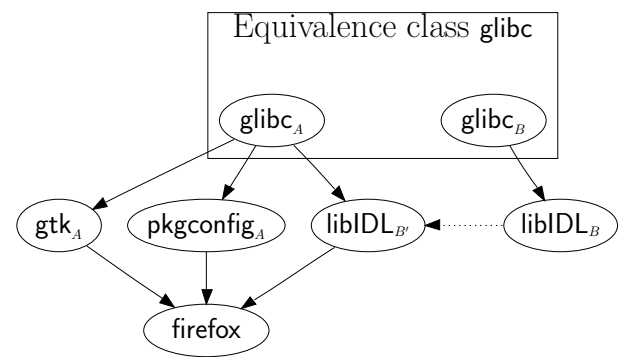

Figure 7: Resolution of the equivalence class collision

Once again, hash rewriting comes to the rescue. We can rewrite $\operatorname{libIDL}_{B}$ so that it refers to glibc instead of glibc $_{B}$. That is, we compute a new path libIDL ${ }_{B}^{\prime}$ with contents

$$
\begin{aligned}
& \text { subst (contents }\left(\operatorname{libIDL}_{B}\right), \\
& \left.\quad\left\{\operatorname{hashPart}\left(\text { glibc }_{B}\right) \rightsquigarrow \operatorname{hashPart}\left(\text { glibc }_{A}\right)\right\}\right)
\end{aligned}
$$

(Of course, self-references in libIDL ${ }_{B}$ must also be rewritten as described in Section 4.2.) This is shown in Figure 7. The dotted edge denotes a copy-with-rewriting action.

An interesting problem is which paths to select from each equivalence class such that the number of rewrites is minimised. For instance, if we select glibc ${ }_{A}$, then we have to rewrite one path (namely liblDL $L_{B}$ ), while if we select glibc $c_{B}$, we have to rewrite two paths ( gtk $_{A}$ and pkgconfig ${ }_{A}$ ). I do not currently know whether there exists an efficient algorithm to find an optimal solution. A heuristic that works fine in practice is to do a bottom-up traversal of the equivalence class dependency graph, picking from each class the path that induces the least number of rewrites.

However, picking an optimal solution with respect to the current derivation is not particularly useful in any case, since it ignores both the state of the Nix store as a whole, and future derivations. For instance, in our example Alice might in the future install many additional components from Bob's remote repository (e.g., because Bob is a primary distribution site). Thus, globally there are many more paths referring to glibc $c_{B}$ than to glibc $c_{A}$. In this case it is better to select glibc $c_{B}$ and rewrite Alice's locally built components. Good heuristics include selecting the path that has the largest number of references to it, or the path that is also trusted by the system administrator (e.g., a special user named root).

Figure 8 shows the resolution algorithm. The function resolve accepts a set of pairs $(p, e)$ each denoting a path $p$ in an equivalence class $e$. This set is closed under the references relation but possibly violates the uniqueness invariant. The function yields a set of paths that does obey the invariant. The function selectPaths must implement some policy of selecting a path from each equivalence class, as discussed above. It returns a map from equivalence classes to paths, e.g., selected $\left[\mathrm{glibc}_{e q}\right]=\mathrm{glibc}_{A}$ for the example in Figure 7. By definition, the paths in this set meet the uniqueness

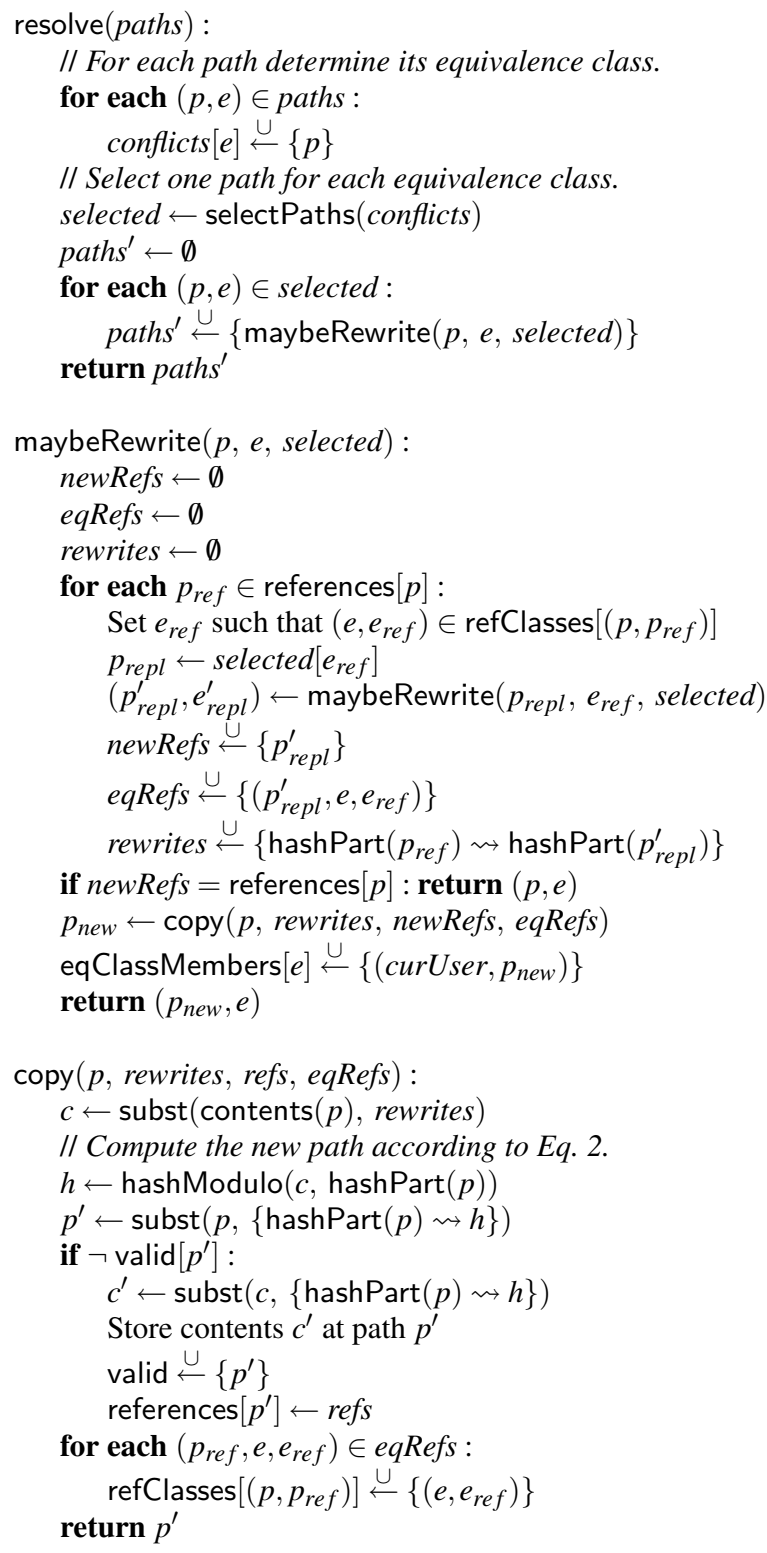

Figure 8: Collision resolution algorithm

invariant, since only one path for each equivalence class is selected. However, they are not necessarily closed, so paths referring to paths outside of the set must be rewritten to refer only to paths in the set. This is done by maybeRewrite, which inspects each reference $p_{r e f}$ of path $p$, maps it onto the equivalent path $p_{\text {repl }}$ in the selected set, and recursively rewrites it into $p_{\text {repl }}^{\prime}$. Then, if any of the references changed, $p$ itself is rewritten. (For brevity, memoisation of maybeRewrite is omitted. Also, we do not show how sources, i.e., pairs $(p, \varepsilon)$ in paths are treated. These are simply left untouched by resolve, i.e., copied to the resulting set paths' ${ }^{\prime}$ )

The auxiliary function copy copies a store path after applying a set of hash rewrites to the contents. This is the only function that adds valid paths to the store in the intensional model. It also set the references and refClasses mapping for the newly created path. The latter are provided by maybeRewrite through the set eqRefs that contains triples $\left(p_{r e f}, e, e_{r e f}\right)$ denoting that the reference link between $p$ and $p_{\text {ref }}$ corresponds to equivalence classes $e$ and $e_{r e f}$. 
$\operatorname{build}(d)$ :

// Note: curUser is the invoking user.

trusted $\leftarrow$ trustedPaths $(d$.eqClass, curUser $)$

for each $p \in$ trusted:

if substitute $(p)$ : return

// Gather all trusted input closures, then resolve.

inputs $\leftarrow \emptyset$

for each $p \in d$.inputsDrvs :

$d^{\prime} \leftarrow \operatorname{readAndParseDrv}(p)$

build $\left(d^{\prime}\right)$

for each $p^{\prime} \in \operatorname{trustedPaths}\left(d^{\prime}\right.$.eqClass, curUser $)$ : inputs $\longleftarrow$ closure $^{\prime}\left(p^{\prime}, d^{\prime}\right.$.eqClass $)$

for each $p \in d$.inputsSrcs :

inputs $\longleftarrow \operatorname{closure}^{\prime}(p, \varepsilon)$

inputs $\leftarrow$ resolve $($ inputs $)$

// Rewrite equivalence classes to real paths.

mapping $\leftarrow \emptyset$

for each $(p, e) \in$ inputs : mapping $\longleftarrow$ $\longleftarrow$ hashPart $(e) \rightsquigarrow \operatorname{hashPart}(p)\}$

Apply rewrites mapping to $d$.env Vars and $d$.builder

// Build in a temporary path.

output $\leftarrow \operatorname{subst}(d$.eqClass,

$\{$ hashPart $(d$.eqClass $) \rightsquigarrow \operatorname{randomHash}()\})$

d.envVars["out"] $\leftarrow$ output

Run $d$.builder in an environment $d$.envVars

refs $\leftarrow$ the subset of inputs referenced in content (output)

output ${ }^{\prime} \leftarrow \operatorname{copy}($ output, refs, $\emptyset$,

$\left\{\left(p_{r}, d\right.\right.$.eqClass,$\left.e_{r}\right) \mid\left(p_{r}, e_{r}\right) \in$ inputs $\wedge p_{r} \in$ refs $\left.\}\right)$

eqClassMembers $[$ d.eqClass $] \longleftarrow\left\{\right.$ (curUser, output $\left.\left.t^{\prime}\right)\right\}$

Figure 9: Build algorithm in the intensional model

\subsubsection{Build algorithm}

Figure 9 shows the build algorithm for the intensional model. We assume that all operations on the Nix store are done by a privileged user on behalf of the actual users, who do not have write access themselves. The main differences with the build algorithm for the extensional model (Figure 5) are as follows. All trusted members of $d$.eqClass can be used as substitutes. Likewise, the closures of all trusted outputs are added to the set of inputs. Equivalence class collisions in that set are resolved. We rewrite occurrences of equivalence classes in the environment variables and builder location to the actual paths selected by trustedPaths and resolve. For the output path, we construct a temporary path equal to $d$.eqClass but with a random hash part. After the build, we copy and rewrite this temporary path to its final, content-addressable location in the Nix store. The temporary path can then be garbage collected.

The omitted function substitute $(p)$ is similar to the one in the extensional model, except that the substitute program produces a temporary path which we then copy to its final location. Also, substitutes are now registered per user, and substitute only tries substitutes registered by the current user. Content-addressability allows the function to verify that a substitute for a path $p$ does indeed create content that matches $p$, i.e., that invariant 3 holds.

\section{THE MIXED MODEL}

The intensional model described in the previous section gives us a Nix store that can be shared by mutually untrusted users, or users who have different remote trust relations. Due to contentaddressability, we get sharing between multiple builds of a derivation if each build produced exactly the same binary result, that is, if there is no impurity in the build. If there is impurity, then each build result will end up under a different store path.

Between untrusted users, this is exactly what we want. For instance, if Alice obtains substitutes from a malicious machine, it does not affect Bob. Note that Alice and Bob do get sharing if they happen to get their substitutes from the same remote machine.

However, we want to re-enable sharing in common scenarios. For instance, users generally trust components installed by the administrator. Thus, if Alice is an administrator, than Bob should be able to use the output paths already installed by Alice. In general, users should be able to specify trust relations between each other.

We can achieve this through a simple extension of the intensional model called the mixed model. For each user, we maintain a mapping trustedUsers : Userld $\rightarrow\{$ Userld $\}$ that specifies for each user a set of trusted users. E.g., trustedUsers["bob"] = $\{$ "alice","bob" $\}$. (The mapping should be reflexive, that is, $u \in$ trustedUsers $[u]$.) We then augment the function trustedPaths:

$$
\begin{aligned}
& \text { trustedPaths }(\text { eqClass, user })= \\
& \quad\{p \mid \exists u \in \text { trustedUsers }[\text { user }]: \\
& \quad(u, p) \in \text { eqClassMembers }[\text { eqClass }]\}
\end{aligned}
$$

Otherwise, this is exactly the intensional model. Of course, sharing between users increases the possibility of equivalence class collisions, but that is handled through the resolution algorithm in Section 4.3.2. The name "mixed model" does not imply that we back away from intensionality — the store is still fully contentaddressed. We just gain back the ability to have sharing between users. The crucial difference with the extensional model is that sharing is now selective and fine-grained.

\section{DISCUSSION}

In this section we briefly discuss our experiences with the implementation of the intensional model, and the security implications of content-addressability through cryptographic hashes.

\subsection{Evaluation}

The most risky part of the intensional model is the use of hash rewriting. It comes as a shock to some that this even works, i.e., doesn't produce broken binaries. In [2], we even wrote that "patching files [by rewriting hashes] is unlikely to work in general, e.g., due to internal checksums on files being invalidated in the process." It turns out that this assessment was too pessimistic. Whether the technique is practical is an empirical question. We have applied hash rewriting to a set of applications from the Nix Packages collection (a large set of Nix expressions for existing Unix software) consisting of 86 components, and verified that the resulting applications were functional. These applications include $\mathrm{C} / \mathrm{Unix}$ programs such as Firefox, as well as Java and C\# programs such as Monodevelop. We encountered no problems.

Hash rewriting necessarily fails in case of pointer hiding [2], i.e., when references to components are stored in such a way that they are not detected as such (e.g., in compressed files). However, this also causes dependency scanning to fail, and those cases are very rare (in fact, to date we have not encountered them at all).

\subsection{Cryptographic hashing}

A content-addressable system depends for its correctness on the assumption that collisions of the hash function being used do not occur in practice. That is, it should be computationally infeasible to produce two inputs that hash to the same value. This is the 
basic goal of cryptographic hash functions [10]. Nix uses 160-bit (truncated) SHA-256 hashes, meaning that the brute-force effort required to find a collision is $2^{80}$ hash operations on average. However, the possibility that cryptographic hash functions might suddenly be broken is a threat to the long-term deployment of tools depending on their security. Indeed, MD5 has recently been broken [16], and SHA-1 weakened [15].

\section{RELATED WORK}

Nix's transparent source/binary model is a unique feature for a deployment system. Relative to binary-only or source-only deployment models, it adds the complication that we do not only need to authenticate binaries but also the fact that they are a bona fide result of certain sources. However, caching and sharing between users of build results is a feature of some SCM systems such as Vesta [7].

As claimed in the introduction, deployment systems tend to have monolithic trust models. For instance, typical Unix package management systems such as RPM [3], Debian APT, or Gentoo Linux [5], allow installation of software by the administrator only; software installed by individual users is not managed by those tools. On the other hand, Mac OS X application bundles may be installed and moved around by any user, but the system does not track dependencies in any way.

Security aspects of deployment have typically focused on ensuring integrity of components in transit (e.g., by using signatures), and on assessing or constraining the impact of a component on the system (e.g., [14]). We have not addressed the issue of ensuring that remote substitutes have not been tampered with (e.g., by a man-in-the-middle). Obviously, such problems can be solved by cryptographically signing substitutes, or rather, the manifests (lists of substitutes available on a server), since the fact that substitutes themselves have not been tampered with is easily verified by comparing their cryptographic hashes to their names.

Microsoft's .NET has a Global Assembly Cache that permits sharing of components [12]. This is however not intended for storage of components private to an application. Thus, if multiple users install an application having such private components, duplication can occur. Also, .NET has a purely binary deployment model, thus bypassing source/binary correspondence issues.

In [6] a scenario is described in which components impersonate other components. This is not possible in a content-addressable file system with static component composition (e.g., Unix dynamic libraries with RPATHs pointing to the full paths of components to link against, as happens in the Nix Packages collection).

Content-addressability is a common property of the distributed hash schemes used in peer-to-peer file-sharing and caching applications (e.g., Pastry [9]). It is also used in the storage layer of version management tools such as Monotone [8].

\section{CONCLUSION}

The Nix deployment system (available at http://nix.cs.uu . $\mathrm{nl}$ ) has many useful properties, such as reliable dependencies, sideby-side deployment of different versions or variants of components, the ability to atomically upgrade or roll back, transparent source/binary deployment, and the ability for different users in a system to independently maintain sets of activated applications. The latter is ideal for multi-user environments, except that Nix's previous $e x$ tensional model required mutual trust between all users. The intensional model described in this paper lifts that requirement, while the mixed model recaptures the sharing of the extensional model using fine-grained trust relations.

In [2] the techniques underlying the Nix system were motivated by analogy to techniques used in programming language implementation. For instance, scanning for hash references in files to determine possible runtime dependencies is analogous to the way conservative garbage collectors find pointers. This paper improves on that result by adding hash rewriting - the analogue of pointer rewriting in copying garbage collectors - to the repertoire of operations in the Nix deployment system.

Acknowledgements This research was supported by CIBIT|SERC and NWO/JACQUARD project 638.001.201, TraCE: Transparent Configuration Environments. I am grateful to Martin Bravenboer and the anonymous referees for their comments.

\section{REFERENCES}

[1] E. Dolstra, M. de Jonge, and E. Visser. Nix: A safe and policy-free system for software deployment. In L. Damon, editor, 18th Large Installation System Administration Conference (LISA '04), pages 79-92, Atlanta, Georgia, USA, November 2004. USENIX.

[2] E. Dolstra, E. Visser, and M. de Jonge. Imposing a memory management discipline on software deployment. In 26th International Conference on Software Engineering (ICSE 2004), pages 583-592. IEEE Computer Society, May 2004.

[3] E. Foster-Johnson. Red Hat RPM Guide. John Wiley and Sons, 2003.

[4] FreeBSD Project. FreeBSD Ports Collection. http://www.freebsd.org/ports/.

[5] Gentoo Foundation. Gentoo Linux. http: //www .gentoo .org/.

[6] M. Grechanik and D. Perry. Secure deployment of components. In W. Emmerich and A. L. Wolf, editors, 2nd International Working Conference on Component Deployment (CD 2004), volume 3083 of Lecture Notes in Computer Science (LNCS). Springer, May 2004.

[7] A. Heydon, R. Levin, and Y. Yu. Caching function calls using precise dependencies. In ACM SIGPLAN 'OO Conference on Programming Language Design and Implementation, pages 311-320. ACM Press, 2000.

[8] G. Hoare. Monotone. http://www . venge . net/monotone/, 2005.

[9] A. Rowstron and P. Druschel. Pastry: Scalable, decentralized object location and routing for large-scale peer-to-peer systems. In 18th IFIP/ACM Intl. Conf. on Distributed Systems Platforms (Middleware 2001), Nov. 2001.

[10] B. Schneier. Applied Cryptography. John Wiley and Sons, second edition, 1996.

[11] W. R. Stevens. Advanced Programming in the UNIX Environment. Addison-Wesley, 1993.

[12] C. Szyperski. Component Software - Beyond Object-Oriented Programming. Addison-Wesley / ACM Press, second edition, 2002.

[13] TIS Committee. Tool Interface Specification (TIS) Executable and Linking Format (ELF) Specification, Version 1.2, May 1995.

[14] V. N. Venkatakrishnan, R. Sekar, T. Kamat, S. Tsipa, and Z. Liang. An approach for secure software installation. In 16th Systems Administration Conference (LISA '02), pages 219-226. USENIX Association, Nov. 2002.

[15] X. Wang, Y. L. Yin, and H. Yu. Finding collisions in the full SHA-1. In CRYPTO 2005, Aug. 2005.

[16] X. Wang and H. Yu. How to break MD5 and other hash functions. In Eurocrypt 2005, May 2005. 